\title{
Considering the cost-effectiveness of statins in family practice in Turkey from a payer perspective
}

\author{
Güvenç Koçkaya ${ }^{1}$, Albert Wertheimer ${ }^{2}$, Pınar Daylan Koçkaya ${ }^{3}$, Ahmet Esen $^{1}$ \\ ${ }^{1}$ Yeditepe Universitesi PEPI, Istanbul, Turkey \\ ${ }^{2}$ Temple University, School of Pharmacy, Philadelphia, USA \\ ${ }^{3}$ Şişli Etfal Education and Research Hospital, Anesthesia Clinic, Istanbul, Turkey
}

Received 2 October 2009; revised 9 November 2009; accepted 10 November 2009.

\begin{abstract}
The percentage of mortality caused by cardiovascular events in European Countries and European Union Countries is respectively $49 \%$ and $42 \%$ of all mortality causes. Our estimates about cardiovascular mortality in Turkey depend on TEKHARF (Hearth Disease and Risk Factors in Turkish Adults) which depended on a 12 year observation. It has been reported that cardiovascular mortality rates for Turkey in men and women were $0.082 \%$ and $0.043 \%$ respectively. In Turkey, Atorvastatin, Fluvastatin, Pravastatin, Rosuvastatin and Simvastatin are the different alternatives found in the statin market. All statins are reimbursed by insurance companies. The aim of this study is to determine the costeffectiveness of statins. In conclusion, simvastatin and rosuvastatin comprised the optimal two statin alternatives.
\end{abstract}

Keywords: Hypercholesterolemia; Cardiovascular Disease; Cost-Effectiveness Analysis; Decision Analysis Mode

\section{BACKGROUND}

The percentage of mortality caused by cardiovascular events in European Countries and European Union Countries is $49 \%$ and $42 \%$, respectively of all mortality causes [1].

Our estimates about cardiovascular mortality in Turkey depend on TEKHARF (Hearth Disease and Risk Factors in Turkish Adults) which was taken from a 12 year observation. In this trial, it was reported that cardiovascular mortality rates for Turkey, in men and women, were $0.082 \%$ and $0.043 \%$ respectively [1].

From this estimation, it can be concluded that every year 140,000 new cardiovascular patients will be diagnosed.

Also in TEKHARF, it was reported that the percent- age of total cholesterol levels above $200 \mathrm{mg} / \mathrm{dL}$ and 249 $\mathrm{mg} / \mathrm{dL}$ were $25 \%$ and $5 \%$ respectively [1].

LDL decreasing and HDL increasing can decrease cardiovascular events. Statins can decrease LDL and increase HDL [2-8]. Some benefits in cardiovascular event protection statin use is increasing $[9,10]$.

In Turkey, there is Atorvastatin, Fluvastatin, Pravastatin, Rosuvastatin and Simvastatin in the statin market. And all statins are reimbursed by health insurance companies [11].

The aim of this study is to consider the cost-effectiveness of statins which are reimbursed by Social Security Foundation from a payer perspective.

\section{METHODS}

A cost-effectiveness analysis was designed from the perspective of the insurance company view. For insurance company data; SSF (Social Security Foundation) which is the biggest reimbursement foundation in Turkey was chosen. The assumed treatment protocol depended on the one in the Republic of Turkey Health Ministry Primary Care Diagnosis and Treatment Guide (THMPCDTG) [12], which was published in 2003. The initial and maintenance doses of statins were taken from Benner [13]. Atorvastatin, Fluvastatin, Pravastatin, Rosuvastatin, Simvastatin initial and maintanence doses were assumed as 10-40-80 $\mathrm{mg} / \mathrm{day}, 40-80 \mathrm{mg} /$ day, $20-40$ $\mathrm{mg} /$ day, $10-20-40 \mathrm{mg} / \mathrm{day}, 20-40-80 \mathrm{mg} /$ day respectively depending on Benner [13] and assumed treatment protocol. The ratios of the effectiveness of the statins which include LDL-C decrease, HDL-C increase and reaching ATP II levels were taken from Benner [13]. The costs of the drugs were taken from Republic of Turkey Drug Pharmacy General Management Drug List; laboratory tests and doctor visits were also taken from the Budget Application Instruction (BAI) [14] from SSF.

As like Benner [13], the analysis employed the payer perspective, hence only direct medical costs, and time horizons of 1 and 3 years (a lifetime analysis was not conducted because longterm clinical data were not yet 
available for all of the treatments used in the model) [13].

In THMPCDTG, it was reported that for starting and maintaining statin treatment it is necessary to know the patients' lipid levels and hepatic enzymes levels. So in each doctor visit these laboratory tests will be repeated. On the other hand, it is also essential to know the creatinine phosphokinase levels only before initiating the treatment. Also in the first 3 months visiting the doctor is essential every 6 weeks and every 3 months in the first year. After the first year the need to visit the doctor is every 6 months [12]. In our assumed treatment protocol depending on THMPCDTG, we assumed that the treatment initiates with initial dosages. In the second visit (6th week), daily dosage will be titrated to half of maximum dosage depending on half of reach ATP II levels and remaining patients will still take the initial dosage. In the third visit (3rd month), dosage will be titrated to maximum dosage depending on ATP II levels and initial dosage and half of the maximum dosage will be administered to the remaining patients too. Our treatment protocol was planned as one year because in the Benner [13] trial the effectiveness of statins was described from the 52-weeks' follow up and estimated as 3 years. The treatment protocol is given in Figure 1. After 52 weeks, last titrated dosages of all statins was assumed as taken following 2 year and for each year 5\% decrease was taken account in drugs and laboratories costs. Also practitioner visits was assumed every 6 month in following second and third years and visits costs was assumed increasing 5\% each year.

The data for patient population and effectiveness of statins was taken from Benner [13]. In this trial, the results of two phase III, randomized clinical studies conducted in Europe and America (Olsson [15] and Brown [16]) were pooled.

A total of 515 patients (atorvastatin $n=116$, pravastatin $n=95$, rosuvastatin $n=202$, simvastatin $n=102$ ) were included in this pooled analysis. $40 \%$ of the patients were male, the mean age was 58, the mean LDL baseline value was $189 \mathrm{mg} / \mathrm{d} \mathrm{L}$ and $23 \%$ of the patients were in a high risk group depending on ATP II criteria.

\subsection{Statin Effectiveness}

The values of the mean effectiveness of statins are shown in Table 1 depending on Benner [13]. In this cohort meta analysis 52 week effectiveness of atorvastatin, pravastatin, rosuvastatin and simvastatin was calculated. Because there was no information about fluvastatin and its effectiveness in Olsson [15], Brown [16] number from Benner's [13] analysis dosage of fluvastatin was considered from the same effectiveness value of provastatin which doubles the potential of fluvastatin. Provastatin was used 20-40 mg daily in the Brown [15] trial so fluvastatin was assumed as 40-80 mg daily.

\section{COSTS}

The direct medical costs were used in this trial from the reimbursement organization perspective. For one year of treatment; the costs of statin usage, doctor visits and laboratory tests were added to the calculation: 6 doctor visits, 6 lipid tests (LDL-K and HDL-K), 6 liver function tests (AST-ALT); and 1 creatinine phosphokinase test were added to the calculation depending on assumed treatment protocol shown in Figure 1. The cost of doctor visits and laboratory tests was taken from SSF's BAI for the year 2009 which was published on www.bumko.gov.tr. The costs of the drugs were taken from Drug Pharmacy General Management Drug List of the Republic of Turkey which was published on 01.06.2009 on www.iegm.gov.tr. In Turkey, each statin has original and generic brands. Atorvastatin, pravastatin, rosuvastatin and simvastatin have 12, 1, 3, 4 generic brands respectively. Fluvastatin does not have any generic version: there is only an original brand. The daily treatment cost of each dosage of each statin which was used in analysis was pooled by using original and generics costs of daily treatments. In this calculation, maximum package containers were chosen for the cost of each dosage. The simvastatin 80 mg dosage form was not available in Turkey's market so it was calculated to use the doubled $40 \mathrm{mg}$ dosage form daily. Then, all costs were changed into US Dollar with $\mathrm{TL} / \mathrm{USD}$ rate as 1.50 , and calculated in USD.

Compliance with statin therapy was assumed to be $100 \%$ in the base-case analysis as Benner [13], because this study pertained only to patients who completed 52 weeks of follow-up, and because differences in adherence between the statins have not been documented [13].

In calculation the results were rounded. The cost added to calculation is shown in Table 2 and Table 3.

\subsection{Alternative Scenarios}

In addition, the impacts of two alternative scenarios that may be reflective for the actual practice were also studied. In the first scenario, it was assumed that patients were not titrated to goal, but they completed the year with initial statin doses. In the second scenario, the patients started treatment with the maximum dosage and completed the year with maximum dose.

\subsection{Adverse Events and Long-Term Outcomes}

Adverse events were not calculated in the model because available evidence suggests that treatmentlimiting event rates do not differ significantly between the statins [13]. Moreover, the average cost of adverse events would be low, because most events resolve after discontinuation of the drug [13]. 
Table 1. Statin effectiveness parameters under Benner study base case.

\begin{tabular}{cccc}
\hline Statin & $\begin{array}{c}\text { LDL-C da \% } \\
\text { Decrease(mean) }\end{array}$ & $\begin{array}{c}\text { HDL-C da \% } \\
\text { Increase (mean) }\end{array}$ & $\begin{array}{c}\text { Reach ATP II } \\
\text { Levels \% (mean) }\end{array}$ \\
\hline Atorvastatin 10-80 mg & 38 & 0.9 & 80 \\
Fluvastatin 40-80 mg & 30 & 4.4 & 60 \\
Pravastatin 20-40 mg & 30 & 4.4 & 60 \\
Rosuvastatin 10-40 mg & 46 & 7.3 & 87 \\
Simvastatin 20-80 mg & 37 & 6.1 & 73 \\
\hline
\end{tabular}

ATP II, National Cholesterol Educaiton Program, Second Adult Treatment Panel; HDL-C, high density lipoprotein cholesterol; LDL-C, low density lipoprotein cholesterol.

Table 2. Cost parameters (In 2009 US dollars and TL).

\begin{tabular}{lcc}
\hline & BAI Codes & Cost \\
\hline Doctor Visit & 520080 & $6,3 \$(9.5 \mathrm{TL})$ \\
Lipid Tests (LDL-K, HDL-K) & 902290,901580 & $2,7 \$(4,10 \mathrm{TL})$ \\
Liver Function Tests (AST-ALT) & 900200,900580 & $1,4 \$(2,10 \mathrm{TL})$ \\
Creatinin Fosfokinaze Test & 902180 & $0,7 \$(1,10 \mathrm{TL})$ \\
\hline
\end{tabular}

Table 3. Cost of daily statin treatments.

\begin{tabular}{|c|c|c|c|}
\hline & & Range & Mean \\
\hline \multirow{3}{*}{ Atorvastatin } & $10 \mathrm{mg} 90$ Tablets ( 1 original +10 generics) & $0,52-0,62 \$(0,79-0,93 \mathrm{TL})$ & $0,57 \$(0,86 \mathrm{TL})$ \\
\hline & $40 \mathrm{mg} 90$ Tablets ( 1 original +10 generics $)$ & $1,18-1,33 \$(1,77-1,99 \mathrm{TL})$ & $1,21 \$(1,82 \mathrm{TL})$ \\
\hline & $80 \mathrm{mg} 90$ Tablets ( 1 original +6 generics) & $1,16-1,39 \$(1,75-2,08 \mathrm{TL})$ & $1,24 \$(1,87 \mathrm{TL})$ \\
\hline \multirow{2}{*}{ Fluvastatin } & 40 mg 28 Tablets ( 1 original) & $0,78 \$(1,17 \mathrm{TL})$ & $0,78 \$(1,17 \mathrm{TL})$ \\
\hline & 80 mg 28 Tablets ( 1 original) & $0,85 \$(1,28 \mathrm{TL})$ & $0,85 \$(1,28 \mathrm{TL})$ \\
\hline \multirow{2}{*}{ Pravastatin } & $20 \mathrm{mg} 20$ Tablets ( 1 original +1 generic) & $0,36-0,45 \$(0,54-0,68 \mathrm{TL})$ & $0,41 \$(0,61 \mathrm{TL})$ \\
\hline & $40 \mathrm{mg} 30$ Tablets ( 1 original +1 generic) & $0,72-0,85 \$(1,09-1,28 \mathrm{TL})$ & $0,79 \$(1,18 \mathrm{TL})$ \\
\hline \multirow{3}{*}{ Rosuvastatin } & $10 \mathrm{mg} 90$ Tablets ( 1 original +1 generic) & $0,75-0,85 \$(1,12-1,28 \mathrm{TL})$ & $0,80 \$(1,20 \mathrm{TL})$ \\
\hline & $20 \mathrm{mg} 90$ Tablets ( 1 original +1 generic) & $1,10-1,43 \$(1,66-2,14 \mathrm{TL})$ & $1,26 \$(1,90 \mathrm{TL})$ \\
\hline & 40 mg 90 Tablets ( 1 original) & $1,10 \$(1,66 \mathrm{TL})$ & $1,10(1,66 \mathrm{TL})$ \\
\hline \multirow{3}{*}{ Simvastatin } & $20 \mathrm{mg} 28$ Tablets ( 1 original +4 generic) & $0,13-0,16 \$(0,20-0,25 \mathrm{TL})$ & $0,14 \$(0,21 \mathrm{TL})$ \\
\hline & $40 \mathrm{mg} 28$ Tablets ( 1 original +4 generic) & $0,27-0,33 \$(0,47-0,50 \mathrm{TL})$ & $0,28 \$(0,43 \mathrm{TL})$ \\
\hline & $80 \mathrm{mg}(40 \mathrm{mg} 28$ Tablets $\times 2)(1$ original +4 generic $)$ & $0,54-0,66 \$(0,94-1 \mathrm{TL})$ & $0,56 \$(0,86 \mathrm{TL})$ \\
\hline
\end{tabular}

Also excluded were costs for future clinical outcomes such as myocardial infarction, stroke, coronary artery bypass grafting or percutaneous transluminal coronary angioplasty, even though statin therapy has been shown to reduce the frequency of these procedures [13].

Excluding these potential cost offsets is consistent with the short-term time frame of the analysis and gives a more conservative estimate of the cost-effectiveness of statin therapy, particularly among the most effective statins [13].

Short-term time frame of the analysis let us make an analysis for family practitioners. Because if patients can't reach optimum cholesterol levels, they need to go specialist practitioners like general medicine, cardiology, etc.

\section{RESULTS}

\subsection{Base Case Analysis}

In the base case analysis, the mean reductions in LDL-C for atorvastatin, fluvastatin, pravastatin, rosuvastatin, simvastatin were $38,30,30$, and 46 respectively. Simvastatin had the lowest cost in the first year of therapy (\$166), followed by pravastatin (\$300), fluvastatin $(\$ 365)$, rosuvastatin $(\$ 437)$ and atorvastatin (\$448). When the drugs were compared for the incremental costeffectiveness, simvastatin dominated pravastatin and fluvastatin, whereas rosuvastatin dominated atorvastatin (Tables 4-6). The first year incremental cost of rosuvastatin was $\$ 271$ compared with simvastatin, or $\$ 30$ per additional $1 \%$ reduction in LDL-C, $\$ 225$ per additional $1 \%$ increase in HDL-C and $\$ 1856$ per additional patients to ATP II goal.

When the drugs were compared for cost per HDL-C increase and LDL-C decrease simvastatin had the least costs for both criteria (27 and 4, respectively), followed by rosuvastatin (60 and 9 , respectively), pravastatin (68 and 10 , respectively), fluvastatin ( 83 and 12 , respectively) and atorvastatin (497 and 12, respectively) (Table 7).

All the dosages of Simvastatin had lower acquisition 
Table 4. 1 st year base case cost per $1 \%$ reduction in LDL-C in Benner study.

\begin{tabular}{|c|c|c|c|c|c|c|c|c|c|}
\hline \multirow[t]{2}{*}{ Strategy } & \multicolumn{2}{|c|}{ Average cost $(\$)$} & \multicolumn{2}{|c|}{ Incremental Cost (\$) } & \multirow[t]{2}{*}{$\begin{array}{l}\text { Average } \\
\% \text { LDL-C }\end{array}$} & \multicolumn{2}{|c|}{ Incremental \% LDL-C $\downarrow$} & \multicolumn{2}{|c|}{$\begin{array}{c}\text { Incremental } \\
\text { cost-effectiveness ratio } \\
(\$ / \mathrm{I} \% \text { LDL-C } \downarrow)\end{array}$} \\
\hline & 1 Year & 3 Years & 1 Year & 3 Years & & 1 Year & 3 Years & 1 Year & 3 Years \\
\hline Simvastatin & 166 & 417 & - & - & 37 & - & - & - & - \\
\hline Pravastatin & 300 & 802 & Dominated & Dominated & 30 & Dominated & Dominated & Dominated & Dominated \\
\hline Fluvastatin & 365 & 970 & Dominated & Dominated & 30 & Dominated & Dominated & Dominated & Dominated \\
\hline Rosuvatatin & 437 & 1189 & 271 & 772 & 46 & 9 & 9 & 30 & 85 \\
\hline Atorvatatin & 448 & 1220 & Dominated & Dominated & 38 & Dominated & Dominated & Dominated & Dominated \\
\hline
\end{tabular}

Cost effectiveness ratios were calculated before cost and effectiveness estimates were rounded. LDL-C, low density lipoprotein cholesterol.

Table 5. 1 year Benner study's base case cost per 1\% increase in HDL-C.

\begin{tabular}{|c|c|c|c|c|c|c|c|c|c|}
\hline \multirow[t]{2}{*}{ Strategy } & \multicolumn{2}{|c|}{ Average Cost(\$) } & \multicolumn{2}{|c|}{ Incremental Cost (\$) } & \multirow[t]{2}{*}{$\begin{array}{l}\text { Average } \\
\% \text { HDL-C }\end{array}$} & \multicolumn{2}{|c|}{ Incremental \% HDL-C } & \multicolumn{2}{|c|}{$\begin{array}{c}\text { Incremental } \\
\text { cost-effectiveness ratio } \\
(\$ / \mathrm{I} \% \text { HDL-C } \mathbf{Y})\end{array}$} \\
\hline & 1Year & 3 Years & 1 Year & 3 Years & & 1 Year & 3 Years & 1 Year & 3 Years \\
\hline Simvastatin & 166 & 417 & - & - & 6.1 & - & - & - & - \\
\hline Pravastatin & 300 & 802 & Dominated & Dominated & 4.4 & Dominated & Dominated & Dominated & Dominated \\
\hline Fluvastatin & 365 & 970 & Dominated & Dominated & 4.4 & Dominated & Dominated & Dominated & Dominated \\
\hline Rosuvastatin & 437 & 1189 & 271 & 772 & 7.3 & 1.2 & 1.2 & 225 & 643 \\
\hline Atorvastatin & 448 & 1220 & Dominated & Dominated & 0.9 & Dominated & Dominated & Dominated & Dominated \\
\hline
\end{tabular}

Cost effectiveness ratios were calculated before cost and effectiveness estimates were rounded. HDL-C, high density lipoprotein cholesterol.

Table 6. 1 year Benner study's base case cost per patients to ATP II goal*.

\begin{tabular}{|c|c|c|c|c|c|c|c|c|c|}
\hline \multirow[t]{2}{*}{ Strategy } & \multicolumn{2}{|c|}{ Average Cost (\$) } & \multicolumn{2}{|c|}{ Incremental Cost (\$) } & \multirow[t]{2}{*}{$\begin{array}{l}\text { Average } \\
\text { Patients } \\
\text { to ATP } \\
\text { II Goal }\end{array}$} & \multicolumn{2}{|c|}{$\begin{array}{l}\text { Incremental Patients to } \\
\text { ATP II Goal }\end{array}$} & \multicolumn{2}{|c|}{$\begin{array}{c}\text { Incremental } \\
\text { cost-efectiveness ratio } \\
\text { (\$/patients to ATP II } \\
\text { goal) }\end{array}$} \\
\hline & 1 Year & 3 Years & 1 Year & 3 Years & & 1 Year & 3 Years & 1 Year & 3 Years \\
\hline Simvastatin & 166.000 & 417.000 & - & - & 725 & - & - & - & - \\
\hline Pravastatin & 300.000 & 802.000 & Dominated & Dominated & 600 & Dominated & Dominated & Dominated & Dominated \\
\hline Fluvastatin & 365.000 & 970.000 & Dominated & Dominated & 600 & Dominated & Dominated & Dominated & Dominated \\
\hline Rosuvastatin & 437.000 & 1.189 .000 & 271.000 & 772.000 & 871 & 146 & 146 & 1856 & 5287 \\
\hline Atorvastatin & 448.000 & 1.220 .000 & Dominated & Dominated & 800 & Dominated & Dominated & Dominated & Dominated \\
\hline
\end{tabular}

*Assuming 1,000 patients treated with each statin

Cost effectiveness ratios were calculated before cost and effectiveness estimates were rounded.

ATP II, National Cholesterol Education Program ,Second Adult Treatment Panel

Table 7. 1 year Benner study's base case cost per 1\% HDL-C increase and 1\% LDL-C decrease.

\begin{tabular}{|c|c|c|c|c|c|c|c|c|}
\hline \multirow[t]{2}{*}{ Strategy } & \multicolumn{2}{|c|}{$\begin{array}{l}\text { Average } 1 \text { Year } \\
\text { Cost }(\$)\end{array}$} & \multirow{2}{*}{$\begin{array}{c}\text { Average } \\
\% \text { HDL-C } \\
\text { (1 and } 3 \text { Years })\end{array}$} & \multicolumn{2}{|c|}{$\begin{array}{l}\text { Cost per \% HDL-C } \\
\text { increase (\$) }\end{array}$} & \multirow{2}{*}{$\begin{array}{c}\text { Average } \\
\% \text { LDL-C } \downarrow \\
\text { (1 and } 3 \text { Years) }\end{array}$} & \multicolumn{2}{|c|}{ Cost per \%LDL-C decrease (\$) } \\
\hline & 1 Year & 3 Years & & 1 Year & 3 Years & & 1 Year & 3 Years \\
\hline Simvastatin & 166 & 417 & 6.1 & 27 & 68 & 37 & 4 & 11 \\
\hline Pravastatin & 300 & 802 & 4.4 & 68 & 182 & 30 & 10 & 26 \\
\hline Fluvastatin & 365 & 970 & 4.4 & 83 & 220 & 30 & 12 & 32 \\
\hline Rosuvastatin & 437 & 1189 & 7.3 & 60 & 162 & 46 & 9 & 25 \\
\hline Atorvastatin & 448 & 1220 & 0.9 & 497 & 1355 & 38 & 12 & 32 \\
\hline
\end{tabular}

Cost effectiveness ratios were calculated before cost and effectiveness estimates were rounded. LDL-C, low density lipoprotein cholesterol. HDL-C, high density lipoprotein cholesterol. 


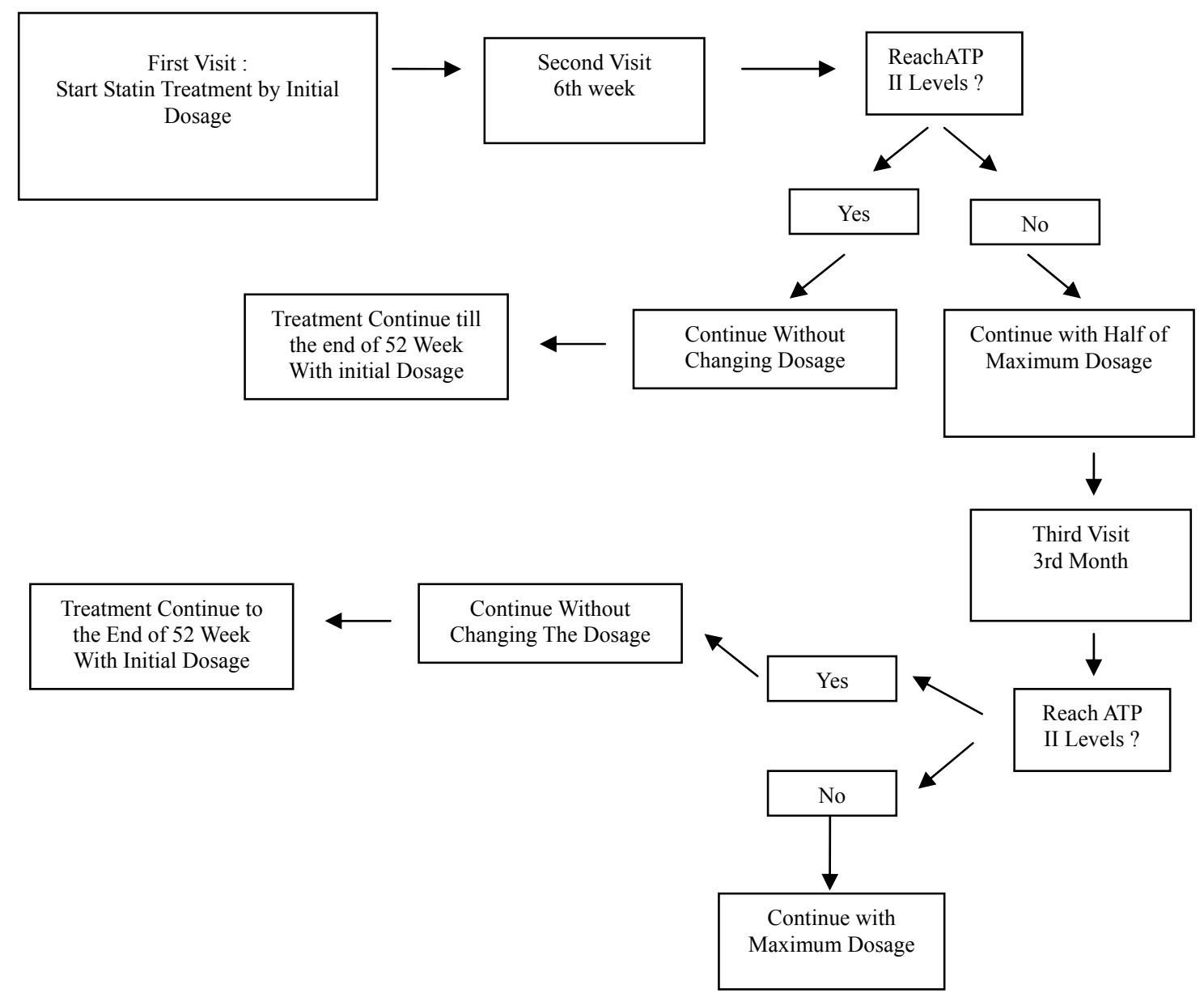

Figure 1. Treatment Protocol : Treatment starts with starting dosage.(Daily Atorvastatin $10 \mathrm{mg}$, Fluvastatin $40 \mathrm{mg}$, Pravastatin $20 \mathrm{mg}$, Rosuvastatin $10 \mathrm{mg}$, Simvastatin $20 \mathrm{mg}$ ). In the 6th week dosage will be titrated to half of the maximum dosage depending on ATP II levels (Daily Atorvastatin $40 \mathrm{mg}$, Fluvastatin $40 \mathrm{mg}$, Pravastatin $20 \mathrm{mg}$, Rosuvastatin $20 \mathrm{mg}$, Simvastatin $40 \mathrm{mg}$ ). In the 3rd month dosage will be titrated to maximum dosage depending on reach ATP II levels (Daily Atorvastatin $80 \mathrm{mg}$, Fluvastatin $80 \mathrm{mg}$, Pravastatin $40 \mathrm{mg}$, Rosuvastatin $40 \mathrm{mg}$, Simvastatin $80 \mathrm{mg}$ ).

cost than all other statins. At initial dosage the acquisition cost is $1 / 3$ of its nearest alternative. So this situation affects the analysis because none of the statins had a double or triple effect in all goals when compared with other statins.

\subsection{Alternative Scenarios}

When the patients were assumed to remain at their respective initial doses and 12 week effectiveness persisted for the full year, again simvastatin (\$116) remained the least costly alternative, followed by pravastatin (\$215), fluvastatin (\$349), rosuvastatin (\$357) and atorvastatin (\$379).

When the patients were assumed to remain at their respective maximum doses and 12 week effectiveness persisted for the full year, again simvastatin (\$269) remained the least costly alternative, followed by pravas- tatin (\$353), fluvastatin (\$375), rosuvastatin (\$488) and atorvastatin (\$517).

As like Benner [13], when the base-case scenario was evaluated using a 3-year time horizon, total costs increased to reflect longer-term statin use (Tables 4-7). Nevertheless, effectiveness was the same as in the 1-year analysis because under recommended monitoring and titration intervals, all titrations occur within the first year of treatment [13]. Thus, the ICERs in the 3-year analysis may be interpreted as the cost to maintain a given level of effectiveness for 3 years [13].

\section{DISCUSSION}

It was reported that statins have a role in decreasing cardiovascular risk in some trials [17]. Also it was reported if $12 \mathrm{mg} / \mathrm{dL}$ decrease occurs in LDL-C levels, cardio- 
vasculer risk increases by $36 \%$ [1].

In this CEA, currently available statins in Turkey in patients with dyslipidemia from perspective of managed care payer were compared.

Because simvastatin had a lower acquisition cost than all statins and its all dosages cost approximately $1 / 3$ of the nearest alternative statin, in our base case and alternative scenarios simvastatin was the least costly alternative.

Simvastatin dominated pravastatin and fluvastatin, whereas rosuvastatin dominated over atorvastatin. Compared with simvastatin, the incremental cost of rosuvastatin was $\$ 271$, or $\$ 30$ per additional $1 \%$ reduction in LDL-C, \$225 per additional 1\% increase in HDL-C and $\$ 1856$ per additional patient to ATP II goal.

Also simvastatin served the least cost for per $1 \%$ decrease in HDL-C and 1\% increase LDL-C, followed by rosuvastatin. For per 1\% HDL-C increase, need to pay for simvastatin and rosuvastatin; $27 \$$ and $60 \$$, respectively. For per $1 \%$ LDL-C decrease need to pay for simvastatin and rosuvastatin; $4 \$$ and $9 \$$, respectively.

These findings have potentially important implications for managed care decision-makers. In the light of the Benner [13] study base case and in each alternative scenario, pravastatin, fluvastatin and atorvastatin always dominated. Thus depending on actual acquisition prices and following costs such as doctor visits and laboratories the payer may achieve substantial cost savings and greater effectiveness by using rosuvastatin or simvastatin instead of these agents in Turkey.

In order to use these findings in a decision-making context, the analysis may be recalculated without including rosuvastatin. The most effective alternative is atorvastatin, which currently is in many formularies. Under base-case assumptions atorvastatin, in the absence of rosuvastatin, has an incremental cost per patient to reach goal of $\$ 75$ when compared with simvastatin.

These findings may have some limitations. Because the objective was to identify the optimal combination of statins for a managed care formulary, several nonstatin medications for dislipidemia (e.g., niacin, fibrates and bile sequestrants) were excluded from the analysis [13]. These products generally have a secondary role in therapy, relatively inexpensive, and were not expected to influence the relative cost-effectiveness of products in the statin class [13]. Their exclusion from this analysis would not be construed to mean that they are not cost-effective agents in modifying serum lipids [13]. The effectiveness of atorvastatin, pravastatin, rosuvastatin and simvastatin were estimated based on data from two phase III trials [13]. Fluvastatin was also assumed to be equivalent to pravastatin over the dose ranges specified, because long-term trials have compared fluvastatin with rosuvastatin [13]. Although the results of the estimates for each drug are consistent with numerous other trials and product labels, and these estimates were varied in sensivity analyses, the results may not be applied to patient populations with different characteristics than those in the Olsson [15] and Brown [16] trials [13]. Moreover, for the detailed analysis for managed care perspective, it is essential to study the effectiveness of statins in Turkish patients. The cost associated with adverse drug events and non-study medications were excluded, but these were not expected to differ across treatment groups, and therefore would not have affected the incremental analysis [13].

In conclusion, the findings of this analysis indicate that simvastatin is a less costly alternative than other statins and rosuvastatin is more effective than other statins. Therefore, simvastatin and rosuvastatin comprise of the optimal two statin formulary. Formulary desicion based on these results should be revisited periodically, as new pricing, outcomes and safety data become available.

\section{ACKNOWLEDGEMENTS}

Thanks Mrs. Sibel Daylan (MS) from Izzet Baysal University and Mr. Ruhi Kadaifci (MD) from Medvice Medical Consultansy for their great help.

\section{REFERENCES}

[1] Turkish Society of Cardiology [National Cardiology Health Politics], Turkish Society of Cardiology Antalya, (2007) www.tkd.org.tr.

[2] The Scandinavian Simvastatin Survival Study Group (1994) Randomised trial of cholesterol lowering in 4444 patients with coronary heart disease: The Scandinavian Simvastatin Survival Study (4S). Lancet, 344, 1383-9.

[3] Shepherd, J., Cobbe, S.M., Ford, I., Isles, C.G., Lorimer, A.R., MacFarlane, P.W., et al. (1995) for the West of Scotland Coronary Prevention Study Group. Prevention of coronary heart disease in men with hypercholesterolemia. N. Engl. J Med, 333, 1301-7.

[4] Sacks, F.M., Pfeffer, M.A., Moye, L.A., Rouleau, J.L., Rutherford, J.D., Cole, T.G., et al. (1996) for the Cholesterol and Recurrent Events Trial Investigators. The effect of pravastatin on coronary events after myocardial infarction in patients with age cholesterol levels. $N$ Engl J Med, 335, 1001-9.

[5] The Long-Tem Intervention with Pravastatin in Ischaemic Disease (LIPID) Study Group (1998) Prevention of cardiovascular events and death with pravastatin in patients with coronary heart disease and a broad range of initial cholesterol levels. $N$ Engl J Med, 339, 1349-57.

[6] Downs, J.R., Clearfield, M., Weis, S., Whitney, E., Shapiro, D.R., Beere, P.A., et al. (1998) for the AFCAPS/TexCAPS Research Group. Primary prevention coronary events with lovastatin in men and women with average cholesterol levels: Results of AFCAPS/TexCAPS. JAMA, 279, 1615-22.

[7] Heart Protection Study Collaborative Group (2002) MRC /BHF heart protection study of cholesterol lowering with simvastatin in 20,536 high risk individuals: A randomised plcebo-controlled trial. Lancet, 360, 7-22. 
[8] Serruys, P.W., de Feyter, P., Macaya, C., Kokott, N., Puel, J., Vrolix, M., et al. (2002) Fluvastatin for prevention of cardiac events following succesful first percutaneous coronary intervention. JAMA, 287, 3215-22.

[9] Jackevicius, C.A., Anderson, G.M., Leiter, L. and Tu, J.V. (2001) Use of the statins in patients after acute myocardial infarction: Does evidence chance practice? Arch Intern Med, 161, 183-8.

[10] Lemaitre, R.N., Furberg, C.D., Newman, A.B., Hulley, S.B., Gordon, D.J., Gottdiener, J.S., et al. (1998) Time trends in the use of cholesterol-lowering agents in older adults. Arch Intern Med, 158, 1761-1768.

[11] Turkey Health Ministry Drug Pharmacy General Man agement [Drug Price List], Ankara, (2009) www.iegm.gov.tr.

[12] Turkey Health Ministry [Primary Care Diagnose and Cure Guide], Ankara, (2003) www.saglik.gov.tr.
[13] Benner, J.S., Smith, T.W., Klingman, D., Tierce, J.C., Mullins, C.D., Pethick, N., et al. (2005) Cost effectiveness of rosuvastatin compared with other statins from a managed care perspective value in health, 8(6), 618-628.

[14] Social Security Foundation [Budget Application Instruction], Ankara, (2009) www.bumko.org.tr.

[15] Olsson, A.G., Istad, H., Luurila, O., Ose, L., Stender, S., Tuomilehto, J., et al. (2002) Rosuvastatin Investigators Group. Effects of rosuvastatin and atorvastatin compared over 52 weeks of treatment in patients with hypercholesterolemia, Am Heart J., 144(6), 1044-51.

[16] Blasetto, J.W., Stein, E.A., Brown, W.V., Chitra, R. and Raza, A. (2003) Efficacy of rosuvastatin compared with other statins at selected starting doses in hypercholesterolemic patients and in special population groups, $\mathrm{Am} \mathrm{J}$ Cardiol., 91(5A), 3C-10C.

[17] Boyac1, B. (2008) Cardiometabolic risk management: Statins in the prevention of cardiovascular events, Turkiye Klinikleri J Med Sci, 28(6 Suppl 1):S, 44-7. 